\title{
Consistency of the Canonical Reduction of General Relativity
}

R. ArnowittS. DeserC. W. Misner

Citation: 1, (1960); doi: 10.1063/1.1703677

View online: http://dx.doi.org/10.1063/1.1703677

View Table of Contents: http://aip.scitation.org/toc/jmp/1/5

Published by the American Institute of Physics 


\title{
Consistency of the Canonical Reduction of General Relativity
}

\author{
R. ARNOWITT* \\ Department of Physics, Syracuse University, Syracuse, New York, and Brandeis University, Waltham, Massachusetts \\ S. DESER* \\ Department of Physics, Brandeis University, Waltham, Massachusetts \\ AND \\ C. W. MisNer $†$ \\ Palmer Physical Laboratory, Princeton University, Princeton, New Jersey, and Brandeis University, Waltham, Massachusetts
}

(Received April 22, 1960)

\begin{abstract}
The question of consistency of the canonical reduction of general relativity (obtained by eliminating constraints and also imposing coordinate conditions in the action or generator) is examined. It is shown that the equations of motion obtained from this "reduced" formalism agree with the original Einstein equations. Agreement is also established for the generators of space-time translations. In order to establish consistency, it is necessary to discard certain well-defined divergence terms in the original Lagrangian. These would otherwise appear as nondivergences in the reduced Lagrangian, incorrectly altering the equations.
\end{abstract}

\section{INTRODUCTION}

A $S$ is well known, general relativity differs from all other field theories in that its "stress tensor" vanishes (to within a divergence) as a consequence of the constraint equations of the theory. This singular behavior arises due to the invariance of relativity under general coordinate transformations. Thus the generator of translations with respect to the (arbitrary) time coordinate would indeed be expected to vanish since any apparent "time" translation could be removed by a mere relabeling. Real motion is to be expressed in terms of physically meaningful time and space coordinates which must be functionals of the metric. Therefore, in order to isolate the dynamics of the system (i.e., to obtain the correct nonvanishing stress tensor), one must specify the dynamical variables as functions of those variables being used as coordinates. Such a procedure is equivalent to imposing coordinate conditions; it also involves elimination of redundant variables by means of the constraint equations.

This program has been previously carried out, ${ }^{1}$ and results in a canonical form for the theory, that is, in a "reduced" Lagrangian of the form

$$
\mathscr{L}=\sum_{A=1}^{2} \pi_{A} \partial \phi^{A} / \partial t-\mathcal{H}\left[\pi_{A}, \phi^{A}\right]
$$

where $\phi^{A}, \pi_{A}$ are two independent canonically conjugate pairs of field variables and $\mathfrak{H}$ is the nonvanishing Hamiltonian density. The functions $\phi^{A}$ and $\pi_{A}$, as

\footnotetext{
* Supported in part by the National Science Foundation and Air Force Office of Seientific Research under contract.

† Alfred P. Sloan Research Fellow.

$I$ In text the following papers will be referred to as $I$ through $V$ :

(I) R. Arnowitt and S. Deser, Phys. Rev. 113, 745 (1959);

(II) R. Arnowitt, S. Deser, and C. Misner, ibid. 116, 1322 (1959);

(III) 117, 1595 (1960); (IIIa) present paper; (IV) 118, 1100

(1960); (IVa) Nuovo cimento (to be published); (IVb) Phys.

Rev. (to be published); (IVc) ibid. (to be published); (V) ibid. (to be published).
}

well as those variables being used as the space-time coordinates, were expressed explicitly in terms of the metric field $g_{\mu \nu}$ and its first time derivatives. The form of Eq. (1.1) automatically ensured the internal consistency of the canonical formalism in that the Poisson bracket (P.B.) equations of motion are identical with the Hamilton-Lagrange equations of motion obtained by varying Eq. (1.1). Correspondingly, the canonical momentum $\mathbf{P}^{C}=-\int d^{3} r \Sigma_{\pi_{A}} \nabla \phi^{A}$ arising in the reduced formalism of Eq. (1.1), correctly generates spatial translations there:

$$
\left[f\left(\pi_{A}, \phi^{A}\right), \mathbf{P}^{C}\right]=\nabla f .
$$

The primary consistency check of the canonical formalism, however, lies in the demonstration of agreement between it and the original Einstein equations. In the process of eliminating redundant variables some subtleties arise, which it is the purpose of this note to examine. In particular, we shall verify that indeed this "external" consistency does hold and that the expressions given for energy-momentum correctly generate space-time translations in the chosen coordinate frame. This result is valid also when matter is coupled to the gravitational field (see Appendix).

In the canonical reduction to Eq. (1.1) from the Einstein-Lagrangian, a complication arises not present in analogous situations for particle mechanics or simple field theories: a term which, in the original Lagrangian (or "Hamiltonian") is a pure divergence, may cease to be a divergence upon elimination of the redundant variables and hence may contribute to the equations of motion obtained from the reduced Lagrangian (or Hamiltonian). Consequently, a Lagrangian obtained by substituting solutions from the constraint equations into the original one may give incorrect equations of motion. For example, suppose a theory contained several dynamical variables $\phi_{A}$ and a redundant variable $C$ with the constraint 
equation

$$
\nabla^{2} C=\phi_{1}^{2}
$$

A term $\nabla \cdot \nabla C$ in the original Lagrangian makes no contribution to the equations of motion there, while in the reduced Lagrangian (where the constraints are eliminated), this term would appear as $\phi_{1}^{2}$ and would contribute. Further, the addition of a divergence may correspond merely to the change of position of a derivative in the original Lagrangian. Whether or not such terms should be included in the original Lagrangian is not directly determined by the original field equations. Yet, the decision to keep or drop these divergences can strongly modify the resulting field equations of the reduced system.

In Lagrangians of the form obtained by parameterizing standard field theories (which form includes general relativity as an "already parameterized" case), there is a unique specification of what divergence terms are to be retained. This requirement leads precisely to a form for the field Lagrangian, which is the natural generalization of the particle case. In the next section it will be shown that upon elimination of redundant variables at this point, the correct equations of motion may be obtained from the reduced Lagrangian. In Sec. 3, the consistency of the spatial generators will be demonstrated. We shall show that the expression previously obtained (in III) for the momentum density (by inserting constraints and coordinate conditions into the original generator), differs from the canonical one merely by a divergence in the canonical variables. Finally, some of our results will be used to comment (in Sec. 4) on other techniques of dealing with general relativity.

\section{REDUCTION OF THE LAGRANGIAN}

It was shown in III that the Lagrangian of general relativity, $\mathcal{L}_{G R}=\left(-{ }^{4} g\right)^{14} R$, could be recast into the form ${ }^{2}$

$$
\begin{aligned}
\mathfrak{L}_{G R}=-g_{i j} \partial \pi^{i j} / \partial t & -N_{\mu} R^{\mu} \\
& -2\left[g^{\frac{1}{k}} N_{0}{ }^{i}+N_{j}\left(\pi^{i j}-\frac{1}{2} g^{i j} \pi\right)\right]_{, i},
\end{aligned}
$$

where

$$
R^{0} \equiv-g^{\frac{1}{2}}\left[{ }^{3} R+g^{-1}\left(\frac{1}{2} \pi^{2}-\pi_{i j} \pi^{i j}\right)\right]
$$

and

$$
R^{i} \equiv-2 \pi^{i j} \mid j
$$

are linear combinations of $G_{\mu}^{0}={ }^{4} R^{0}{ }_{\mu}-\frac{1}{2} \delta^{0}{ }_{\mu}^{4} R$. Here

and

$$
N_{0} \equiv\left(-g^{00}\right)^{-\frac{1}{2}}, \quad N_{i} \equiv{ }^{4} g_{0 i}, \quad \pi \equiv \pi^{i j} g_{i j}
$$

$$
\pi^{i j} \equiv\left({ }^{-4} g\right)^{\frac{1}{2}}\left[{ }^{4} \Gamma_{m n}^{0}-g_{m n}{ }^{4} \Gamma_{p q}^{0} g^{p q}\right] g^{m i} g^{n i} .
$$

We use units such that $\kappa \equiv 16 \pi \gamma c^{-4}=1=c$, where $\gamma$ is the Newtonian gravitational constant. Latin indices run from 1 to 3 , Greek from 0 to 3 , and $x^{3}=t$. All tensors and covariant operations are three-dimensional unless specified; ${ }^{3} R$ is the curvature scalar of $g_{i j}$ (not $g_{\mu v}$ ) and $g^{i j}\left(\neq^{4} g^{i j}\right)$ is the matrix inverse to $g_{i j}$. The vertical bar "" indicates covariant differentiation with respect to $g_{i j}$, and $N_{0}$ is treated as a three-scalar. Ordinary differentiation is denoted by a comma or the symbol $\partial_{\mu}$.
This Lagrangian is of the general form ${ }^{3}$

$$
\begin{aligned}
\mathscr{L}=\sum_{I=1}^{N+4} \pi_{I} \partial \phi^{I} / \partial t-N_{\mu} R^{\mu}\left(\phi^{J}, \pi_{J}\right) & \\
& +\left[\mathcal{F}^{i}\left(\pi_{J}, \phi^{J}, N_{\mu}\right)\right]_{, i}
\end{aligned}
$$

which form is also found when matter is coupled to the gravitational field (see IV and V). The same structure arises upon parameterization of standard field theories (e.g., the scalar meson example in III), and is the straightforward generalization of the parameterized particle Lagrangian ${ }^{4}$

$$
L=\sum_{i=1}^{M} p_{i} q^{i \prime}+p_{M+1} q^{M+1^{\prime}}-N R
$$

where the constraint $R$ may be taken as $R \equiv p_{M+1}$ $+\mathscr{K}\left(p_{i}, q_{i}\right)$; a prime denotes a derivative with respect to the (arbitrary) parameter $\tau$ which replaces the time in this formulation. Note that the divergence term of $\mathcal{L}$ in $\mathrm{Eq}$. (2.4) is determined uniquely by the requirement that the $R^{\mu}$ not be functions of the Lagrange multipliers $N_{\mu}$. For example, gradients appearing in $R^{\mu}$ cannot be moved by means of integrations by parts, which would give rise to gradients of $N_{\mu}$ outside the total divergence term; this is not allowed in the standard form of Eq. (2.4).

In the remainder of this section, we shall compute the equations of motion obtained by varying the action

$$
I=\int d^{4} x \mathcal{L}
$$

and we shall insert into these the solutions of the constraint equations and the coordinate conditions. (The term $\mathcal{F}^{j},{ }_{j}$ in $\mathcal{L}$ does not contribute in this analysis.) We will then verify that these equations agree with those obtained by varying the reduced Lagrangian $\mathscr{L}_{R}$; here $\mathscr{L}_{R}$ is that Lagrangian obtained by substituting constraints and coordinate conditions into $\mathscr{L}$ with the divergence $\mathcal{F}^{j}{ }^{j}$ discarded.

The constraint equations $R^{\mu}=0$ result from varying $N_{\mu}$. They state that four of the momenta $\pi_{I}$ are not independent canonical variables, corresponding to the fact that four of the $\phi^{I}$ are not really field variables, but rather physical space-time coordinates. We assume that $\phi^{I}$ and $\pi_{I}$ are so chosen that the coordinate conditions

$$
\phi^{N+\mu}=x^{\mu}
$$

${ }^{3}$ Since constraint equations, by definition, contain no time derivatives, their solutions never eliminate them. Thus a total time derivative in the Lagrangian is harmless and $\pi \partial_{0} \phi$ is equivalent to $-\phi \partial_{0} \pi$ there, either before or after the elimination of redundant variables. For further discussion of the relation of total time derivatives to canonical transformations, see IVa.

4 See, for example, C. Lanczos, The Variational Principles of Mechanics (University of Toronto Press, Toronto, Canada, 1949), or II. 
are physically acceptable. ${ }^{5}$ With the convention that the index $A$ runs from 1 to $N$, we write the solutions of the constraint equations

$$
\delta I / \delta N_{\mu}=R^{\mu}\left(\pi_{N+\mu}, \phi^{A}, \pi_{A}, \phi^{N+\mu}\right)=0,
$$

in the form

$$
\pi_{N+\mu}=T^{0}\left[\phi^{A}, \pi_{A}, \phi^{N+\mu}\right] .
$$

Since $R^{\mu}$ may involve spatial derivatives, we use the functional notation $\mathcal{T}^{N}{ }_{\mu}\left[\phi^{A}, \pi_{A}, \phi^{N+\mu}\right]$ to take into account the possible appearance of operators such as $\left(1 / \nabla^{2}\right)$ in Eqs. (2.8). Indeed, just this circumstance (having no simple analog in particle mechanics) gives rise to the "divergence" problem under discussion here.

Varying $\phi^{A}$ and $\pi_{A}$ in the action integral yields the equations of motion

$$
\begin{aligned}
& \partial \pi_{A}(x) / \partial t=-\int d^{4} y N_{\mu}(y)\left[\delta R^{\mu}(y) / \delta \phi^{A}(x)\right] \\
& \partial \phi^{A}(x) / \partial t=\int d^{4} y N_{\mu}(y)\left[\delta R^{\mu}(y) / \delta \pi_{A}(x)\right] .
\end{aligned}
$$

Since the $R^{\mu}$ contain no time derivatives, $\delta R^{\mu}(y) / \delta \phi^{A}(x)$ will contain $\delta\left(y^{0}-x^{0}\right)$ as a factor; however, spatial derivatives of $\phi^{A}$ in $R^{\mu}$ produce derivatives of delta functions in $\delta R^{\mu} / \delta \phi^{A}$, and thus spatial derivatives of $N_{\mu}$ may appear when the integral in Eq. (2.9a) [or Eq. (2.9b)] is evaluated. The equations

$$
\partial \phi^{N+\mu}(x) / \partial t=\int d^{4} y N_{\alpha}(y)\left[\delta R^{\alpha}(y) / \delta \pi_{N+\mu}(x)\right]
$$

serve to determine the Lagrange multipliers $N_{\mu}$ when the functions $\phi^{N+\mu}$ are specified by the coordinate conditions.

In order to substitute the solution of the constraints, Eq. (2.8), into the dynamic equations (2.9) we expand $R^{\mu}$ in a functional Taylor series ${ }^{6}$ about the point $\pi_{N+\mu}=T^{0}{ }_{\mu}$ (indicated in the following by $\pi=T^{\prime}$ ). The zeroth-order term vanishes and we have

$$
\begin{aligned}
& R^{\mu}(y)=\int d^{4} z\left[\pi_{N+\alpha}(z)-T_{\alpha}^{0}(z)\right] \\
& \times\left[\delta R^{\mu}(y) / \delta \pi_{N+\alpha}(z)\right]_{x=}+\cdots
\end{aligned}
$$

This allows us to compute

$$
\begin{aligned}
\delta R^{\mu}(y) / \delta \phi^{A}(x)= & -\int d^{4} z\left[\delta T_{\alpha}^{0}(z) / \delta \phi^{A}(x)\right] \\
& \times\left[\delta R^{\mu}(y) / \delta \pi_{N+\alpha}(z)\right]_{x=\tau^{+}}+\cdots
\end{aligned}
$$

\footnotetext{
${ }^{6}$ For general relativity, the main requirement is that these conditions are consistent with an asymptotically flat metric at spatial infinity.

V. Volterra, Theory of Functionals (Blackie and Son Ltd., London, 1930), p. 25.
}

and

$$
\begin{aligned}
\delta R^{\mu}(y) / \delta \pi_{A}(x) & =-\int d^{4} z\left[\delta T_{\alpha}^{0}(z) / \delta \pi_{A}(x)\right] \\
& \times\left[\delta R^{\mu}(y) / \delta \pi_{N+\alpha}(z)\right]_{\pi}=\tau^{+}+\cdots
\end{aligned}
$$

where the terms represented by $\cdots$ contain $\left[\pi_{N+\alpha}\right.$ $\left.-T_{\alpha}^{0}\right]$ as a factor and so vanish when the constraints are inserted. We now substitute Eqs. (2.12) into (2.9) and eliminate $\pi_{N+\alpha}$ by using Eqs. (2.8). The coordinate conditions (2.6) reduce Eq. (2.10) to $\delta^{\mu}{ }_{0}=\int N_{\alpha} \delta R^{\alpha} / \delta \pi_{N+\mu}$. By using these results, the dynamic equations then become

$$
\begin{aligned}
& \partial \pi_{A}(x) / \partial t=\int d^{\mathrm{g}} z\left[\delta T^{0} 0_{0}(z) / \delta \phi^{A}(x)\right] \\
& \partial \phi^{A}(x) / \partial t=-\int d^{4} z\left[\delta T^{0}{ }_{0}(z) / \delta \pi_{A}(x)\right] .
\end{aligned}
$$

These equations, equivalent to the original set [Eqs. (2.9) and (2.7)] under the coordinate condition $\partial_{0} \phi^{N+\mu}=\delta^{\mu}{ }_{0}$ are easily seen to be just the Hamiltonian equations obtained from the Lagrangian

$$
\mathfrak{L}_{R}=\sum_{A=1}^{N} \pi_{A} \partial \phi^{A} / \partial t-\mathfrak{K}
$$

where

$$
\mathfrak{K}=-\mathcal{T}^{0}\left[\phi^{A}, \pi_{A}\right] .
$$

Note that only the differential statement of the coordinate conditions, $\partial_{0} \phi^{N+\mu}=\delta^{\mu}{ }_{0}$, was needed in deriving the preceding result. In Eq. (2.14b) we have assumed that these coordinate conditions are chosen in such a way that $q^{* 0}$ has no explicit $x^{s}$ dependence in order that a set of standard conservations laws hold. ${ }^{7}$

For general relativity, one must rearrange the $g_{i j} \partial_{t} \pi^{i j}$ part of the Lagrangian of Eq. (2.1) in order to explicitly apply the general methods discussed above. This is accomplished by making an orthogonal decomposition on $\pi^{j i}$ and $g_{i j}$ similar to the one used in III. We write

$$
\begin{aligned}
& g^{i j}=\delta_{i j}+h_{i j} \\
& =\delta_{i j}+h_{i j}{ }^{T T}+\frac{1}{2}\left[\delta_{i j} h^{T}-\left(1 / \nabla^{2}\right) h^{T}{ }^{T}, i j\right] \\
& +h_{i, j}+h_{j, i} \\
& \pi^{i j}=\pi^{i j T T}+\frac{1}{2}\left[\delta_{i j} \nabla^{2} \bar{\pi}^{T}-\bar{\pi}^{T}, i j\right]+\pi^{i},{ }_{j}+\pi^{j},{ }_{i} .
\end{aligned}
$$

Note that $\nabla^{2} \bar{\pi}^{T}$ used here is just $\pi^{T}$ of III and $h_{i j}$ approaches zero asymptotically. The Lagrangian (2.1) now becomes

$$
\begin{array}{r}
\mathscr{L}=\pi^{i j T^{T} T} \partial_{t} h_{i j}{ }^{T T}+\nabla^{2} h^{T} \partial_{0}\left(-\frac{1}{2} \bar{\pi}^{T}\right)-2 \pi^{i j}{ }_{j} \partial_{0} h_{i} \\
+N_{\mu} R^{\mu}+\mathcal{F}^{\prime j}{ }_{j, j} .
\end{array}
$$

${ }^{7}$ That this can in fact be achieved for general relativity was shown in III. 
In obtaining Eq. (2.16), various spatial divergences have been included in $\mathfrak{F}^{\prime}{ }_{, j}$ and a total time derivative neglected. ${ }^{3}$ By choosing

one has

$$
\phi^{N+0} \equiv-\frac{1}{2} \bar{\pi}^{T}, \quad \phi^{N+i}=x^{i}+h_{i},
$$

$$
\begin{array}{r}
\mathscr{L}=\pi^{i j T T} \partial_{t} h_{i j}{ }^{T T}-\left(-\nabla^{2} h^{T}\right) \partial_{t} \phi^{N+0}-2 \pi^{i j}, \partial_{t} \phi^{N+i} \\
+N_{\mu} R^{\mu}+F^{\prime j}{ }_{, j} .
\end{array}
$$

The formal derivation now follows with the association

$$
\begin{aligned}
\pi_{A} & \equiv \pi^{i j T T}, \quad \phi^{A} \equiv h_{i j}{ }^{T T} \\
\pi_{N+0} & \equiv \nabla^{2} h^{T}, \quad \pi_{N+i} \equiv-2 \pi^{i j}, j,
\end{aligned}
$$

since $\mathcal{L}$ is now in the form of Eq. (2.4). As was discussed in III, the constraint equations $R^{\mu}=0$ can indeed be solved for $\pi_{N+\mu}$ and are independent of $x^{\mu}$ when the coordinate conditions $\phi^{N+\mu}=x^{\mu}$ are imposed.

The divergence $\mathfrak{F}^{j}{ }_{j}$ in Eq. (2.4) played no role whatsoever, as a divergence never affects a variational derivative. However, $\mathfrak{L}_{R}$ of Eq. (2.14) will not necessarily be the reduced Lagrangian obtained by inserting $\mathrm{CO}^{-}$ ordinate conditions (2.6) and constraints (2.8) into the original Lagrangian of Eq. (2.4) unless $\mathcal{F}^{j}, j$ is dropped, as was illustrated by the simple example in Sec. I. (If some particular $\mathfrak{F}^{i}, j$ happens to remain a divergence after $\phi^{N+\mu}, \pi_{N+\mu}$ and $N_{\mu}$ have been eliminated, the reduced Lagrangian will be equivalent to $\mathscr{L}_{R}$, but in general the correct way to obtain $\mathfrak{L}_{R}$ is to drop the $\mathfrak{F}^{j}{ }_{j}$ term before eliminating the redundant variables.) In relativity it is very easy to obtain equations such as $\partial \phi^{A} / \partial t=0=\partial \pi_{A} / \partial l$ by including a wrong divergence term in $\mathfrak{L}$ while substituting constraints. In fact, it is easy to obtain such equations for variables $\phi^{A}$ and $\pi_{A}$, which are not even constants of motion in the linearized approximation.

The result obtained in this section is not an unexpected one. If one drops the term $\Im^{i},{ }_{j}$ in Eq. (2.4) (which does not affect the equations of motion obtained from $\mathscr{L}), \mathcal{L}$ becomes just the field analog of the parameterized particle Lagrangian. It is well known ${ }^{4}$ in the particle mechanics case that one can then impose the constraint and coordinate conditions without error.

\section{REDUCTION OF THE GENERATOR AND FIELD MOMENTUM}

We consider here the generator associated with the Lagrangian of Eq. (2.4). As was discussed in III, the process of reducing the theory to canonical form can be carried out directly in the generator. We will show explicitly that this reduction is identical to the previous one. In addition, it will enable us to display the field momentum density automatically. We will see that this differs from the field momentum density derived from the reduced Lagrangian, Eq. (2.14), by a divergence of canonical variables (a "true divergence"); this will check the consistency of the spatial displacement generators.
The generator arises from the Lagrangian of Eq. (2.4) (with the $\mathcal{F}^{j}{ }_{j}$ now discarded) as the surface integral term in the endpoint variation of the action. It has the form

$$
G=\int d^{3} r\left[\sum_{I=1}^{N+4} \pi{ }^{\prime} \delta \phi^{I}-T^{0}{ }_{\mu}^{\prime} \delta x^{\mu}\right]
$$

The stress tensor $T^{0}{ }_{\mu}^{\prime}$ vanishes (to within a divergence) as a consequence of the constraint equations, which again reflects the general covariance of the theory. Thus, the canonical tensor which arises when one makes arbitrary coordinate variations $\delta x^{\mu}$ in $\mathrm{Eq}$. (2.5) (but no associated Lorentz transformations) is given by

$$
\mathcal{T}^{0}{ }_{0}{ }^{\prime}=N_{\mu} R^{\mu}
$$

and

$$
\mathcal{T}^{0}{ }_{k}{ }^{\prime}=-\sum_{I=1}^{N+4} \pi_{I} \phi^{I}, k
$$

The constraint equations $R^{\mu}=0$ obviously account for the vanishing of $\mathcal{T}_{0}^{0}{ }_{0}$ and we will see that $\mathcal{T}^{0}{ }_{k}^{\prime}$ also vanishes (to within a divergence). The generator therefore becomes

$$
G=\int d^{3} r\left[\sum_{A=1}^{N} \pi_{A} \delta \phi^{A}+\sum_{\mu=0}^{3} \pi_{N+\mu} \delta \phi^{N+\mu}\right] .
$$

On inserting solutions (2.8) of the constraint equations and the coordinate conditions (2.6), one has

$$
G=\int d^{3} r\left[\sum_{A=1}^{N} \pi_{A} \delta \phi^{A}+T^{0}{ }_{\mu} \delta x^{\mu}\right]
$$

which is the standard canonical form for a field theory generator with $N$ canonical pairs of variables $\phi^{A}, \pi_{A}$ and a generator of space-time translations $\int T^{0}{ }_{\mu} \delta x^{\mu} d^{3} r$. The Hamiltonian $\mathfrak{H}=-\int d^{3} r T^{0}{ }_{0}$ is identical to the one obtained in Sec. 2, thus showing the correctness of the time-translation part. From the reduced Lagrangian $\mathscr{L}_{R}$ of Eq. (2.14), one knows that the correct generator of space translations is the canonical field momentum

$$
P_{k}=\int d^{3} r T^{c 0_{k}} \equiv-\int d^{3} r \sum_{A} \pi_{A} \phi^{A}, k=
$$

(By Eq. (3.6) and the fundamental P.B. relations, one has $\left.\left[f, P_{k}\right]=\partial_{k} f\right\}$. From Eq. (3.3), one has

$$
T^{0}{ }_{k}^{\prime}=T^{c 0_{k}}-\sum_{\mu=0}^{3} \pi_{N+\mu} \phi^{N+\mu}, k .
$$

On inserting the solutions of the constraint equations (2.8) and the coordinate conditions (2.6), one obtains

$$
T^{0}{ }_{k}^{\prime}=T^{C 0_{k}}-T_{k}^{0} \text {. }
$$

Thus, the vanishing of $T_{k}^{\prime}$ coincides with the 
consistency requirement for the spatial-translation generators. $^{8}$

We now show that $T^{0}{ }_{k}$ differs from $T^{c 0}{ }_{k}$ by at most a divergence of the canonical variables. We limit ourselves to the case of general relativity. Here $\phi^{A}$ $\equiv h_{i j}{ }^{T T}, \quad \pi_{A} \equiv \pi^{i j T T}, \quad T_{0}{ }_{0}=\nabla^{2} h^{T}, \quad$ and $\quad T^{0}{ }_{i}=-2 \pi^{i j}{ }_{j}$ (notation is as in Sec. 2), while $T^{C 0_{k}}=-\pi^{i j T T} h_{i j}{ }^{T T}{ }_{k}$. The constraint equation which determines $T^{* 0}{ }_{i}$ in terms of the canonical variables reads

$$
-2 \pi^{i i}{ }_{1 j}=0
$$

and can be written as

$$
-2 \pi_{i}^{j}, j \equiv-2\left(g_{i k} \pi^{k j}\right), j=-\pi^{i j} g_{l j, i} .
$$

On inserting the orthogonal decomposition for $\pi^{l j}, g_{l j}$ in Eq. (3.10), one has

$$
\begin{aligned}
-2 \pi_{i}{ }^{j}, j=-\pi^{l j T T} h_{l j}{ }^{T T}{ }_{, i}- & {\left[2 \pi^{l}\left(h_{l j}{ }^{T T}{ }_{, i}+h_{l j}{ }^{T}{ }_{, i}\right)\right.} \\
& \left.-\frac{1}{2} \pi^{l i T T}\left(1 / \nabla^{2}\right) h^{T}, i i\right], j .
\end{aligned}
$$

It is now necessary to show that the []$, j$ term of Eq. (3.11) is indeed a true divergence in the sense that $f[],{ }^{3} d^{3}$ vanishes for arbitrary values of the canonical variables. These latter must vanish rapidly for the system to be bounded (see IVb). When $\pi^{l}$ and $g^{T}$ are expressed in terms of the canonical variables, they are seen to decrease as $1 / r$ at infinity. Thus all the terms in the bracket vanish faster than $1 / r^{2}$, and therefore $-2 \pi_{i}{ }^{i},{ }_{j}$ differs from $T^{C 0}{ }_{i}$ by a true divergence. Further, since

we find

$$
g_{i j}=\delta_{i j}+h_{i j}{ }^{T}+h_{i j}{ }^{T}
$$

$$
-2 \pi_{i}{ }^{j}, j=-2 \pi^{i j}, j+2\left[\pi^{l j}\left(h_{i l^{T}}{ }^{T}+h_{i j}{ }^{T}\right)\right], j,
$$

where the bracket in Eq. (3.12) is also a true divergence. Hence

$$
P_{i}=\int d^{3} r \mathcal{T}^{c 0_{i}}=\int T^{0}{ }_{i} d^{3} r \equiv-2 \int d^{3} r \pi^{i j}, j
$$

This establishes explicitly that the term $\int d^{3} r T^{0}{ }_{i} \delta x^{i}$ arising in Eq. (3.5) correctly generates spatial translations. The result holds also when matter is coupled to the gravitational field, as shown in the Appendix.

Equivalence between $T^{0}{ }_{k}$ and $T^{c 0}{ }_{k}$ is valid for a parameterized Lorentz covariant theory as well. If the parameterization is carried out by rewriting the Lagrangian in a generally covariant form, but with $g_{\mu \nu}=\eta_{\alpha \beta}\left(\partial \phi^{N+\alpha} / \partial x^{\mu}\right)\left(\partial \phi^{N+\beta} / \partial x^{\nu}\right)$ (where $\eta_{\alpha \beta}$ is the Lorentz metric), then one finds for the $T^{0}{ }_{k}$ of Eq. (3.5) the standard symmetric stress tensor of the original theory.$^{9} \mathrm{As}$ is well known, this differs by a divergence from $T^{C{ }^{C O}}{ }_{k}$.

\footnotetext{
${ }^{8}$ Conversely, the physical requirement that $T_{i}^{0}$ must vanish since it is the generator of translations with respect to the parameters (on which the theory does not depend), would then lead to the equivalence of $T^{0_{i}}$ and $T^{C 0}{ }_{i}$.

9 It might be noted that the alternate type of parameterization carried out for the scalar meson field in III, would lead to the canonical tensor for $\boldsymbol{T}^{\mathbf{b}} \boldsymbol{k}$.
}

\section{DISCUSSION}

In the preceding sections we have seen that in a Lagrangian of the form

$$
\mathfrak{L}=\sum_{i=1}^{N+\mu} \pi_{l} \partial \phi^{I} / \partial t-N_{\mu} R^{\mu}\left(\pi_{J}, \phi^{J}\right)
$$

one may insert the solutions of the constraint equations $\pi_{N+\mu}=T^{0}{ }_{\mu}$ and the canonical coordinate conditions $\partial_{\iota} \phi^{N+\mu}=\delta_{0}^{\mu}$ to obtain a reduced Lagrangian

$$
\mathfrak{L}_{R}=\sum_{A=1}^{N} \pi_{A} \partial \phi^{A} / \partial t+\tau^{0} 0\left[\pi_{A}, \phi^{A}\right]
$$

whose equations of motion are equivalent to those of Eq. (4.1). Consequently, given a Lagrangian that differs from Eq. (4.1) by a total 3-divergence, the consistent reduction method requires that this divergence be neglected before eliminating the constraints. In general relativity, then, the last term in Eq. (2.16) should be omitted (as was done in III). Thus, the canonical equations of motion in III are correctly the Einstein equations. Indeed, if one had included the divergence which actually appears in Eq. (3.1), the energy obtained from the reduced Hamiltonian for the Schwarzschild solution would have become $\frac{3}{2} m$ rather than $m$. Similarly, this Hamiltonian would give rise to wrong equations of motion even in the linearized approximation.

It was also seen that the generator associated with the Lagrangian of $\mathrm{Eq}$. (4.1) gives rise, when constraints are eliminated and coordinate conditions imposed, to the generator obtained from the reduced Lagrangian of Eq. (4.2). Thus the consistency of the spatial translation generators is guaranteed.

Recently, Dirac ${ }^{10}$ has suggested an entirely different procedure for obtaining a nonvanishing Hamiltonian in general relativity. His analysis is performed within a generalized Hamiltonian formalism ${ }^{11}$ and does not make direct use of the fact that general relativity is a parameterized theory when presented in generally covariant form. The method involves writing the vanishing Hamiltonian $N_{\mu} R^{\mu}$ as

$$
N_{\mu} R^{\mu}=\left(N_{0}-1\right) R^{0}+N_{i} R^{i}+R^{0},
$$

and dropping a particular divergence in the last term. Thus, before the redundant variables are eliminated, the new Hamiltonian density is weakly a divergence. Next, the redundant variables are eliminated by means of the constraint equations, and one arrives at a reduced Hamiltonian which is not a divergence in terms of the remaining variables. The situation here is just of the type discussed in Eq. (1.3). Such a procedure seems to us to be logically incomplete. While with the

${ }^{10}$ P. A. M. Dirac, Phys. Rev. 114, 924 (1959); Proc. Roy. Soc. (London) A246, 333 (1958); Phys. Rev. Letters 2, 368 (1959).

"P. A. M. Dirac, Proc. Roy. Soc. (London) A246, 326 (1958). 
particular choice of divergence that Dirac makes, the reduced Hamiltonian gives correct equations of motion in the linearized approximation, no general proof is given that the full theory is correct in this respect. It is of interest to note however, that Dirac's choice does lead to the correct numerical value of the energy. ${ }^{12}$

\section{APPENDIX}

Here we extend, for the case of coupling, the proof given in text that the field energy momentum of the reduced generator correctly generates space-time translations. For the momentum, our derivation consists, as in Sec. 3, in showing that this momentum density differs from the canonical one by a true divergence. In $V$, it is shown that when the Maxwell field and point charges are coupled to the gravitational field, the coefficient of $\delta x^{i}$ in the total reduced generator is still $-2 \pi^{i j}, j$. The effect of the matter enters through the constraint equations used to solve for $-2 \pi^{i j}{ }_{, j}$ in terms of the canonical variables of all the fields. Thus, Eq. (3.9) now reads

$$
-2 \pi^{i j}{ }_{\mid j}=g^{i k} T_{M}{ }^{0}{ }_{k}
$$

12 Since Dirac's Hamiltonian differs from zero by a divergence, its numerical value for a computation of the energy is given by this divergence. Thus

$$
E=-\int d^{3} r\left[g^{-i}\left(g g^{i j}\right), i\right], i=-\mathscr{S} g^{-j}\left(g g^{i i}\right), j d S_{i} .
$$

On introducing the orthogonal decomposition of the metric [Eq. (2.15)], we may, in the surface integral at spatial infinity, neglect all terms beyond the linear one since $g_{i j} \rightarrow \delta_{i j}$ at spatial infinity. This gives $E=-\Phi\left[g^{T}, i+\left(g_{i, i}-g_{i, i}\right), j\right] d S_{i}$, but the second term vanishes by Gauss' theorem, leaving

$$
E=-\Phi_{\mathrm{g}}{ }{ }_{i}, d S_{i},
$$

the coordinate independent value obtained in III and IV. This is also equal, as Dirac has noted, to the value obtained from the surface integral form of the Einstein pseudotensor. (This discussion assumes $g_{u n,} \sim 1 / r^{2}$ at spatial infinity; see IVc for a more general treatment.) where the matter momentum density is

$$
T_{M_{k}{ }_{k}}=B_{k j} \mathcal{E}^{j}+\left[p_{k}(t)-e A_{k}{ }^{T}\right] \delta^{3}[\mathbf{r}-\mathbf{r}(t)] .
$$

In Eq. (A2), $B_{k j} \equiv A_{j}{ }^{T}{ }_{, k}-A_{k}{ }^{T}{ }_{, j}$ is the magnetic field, $\mathscr{E}^{j} \equiv \mathcal{F}^{0 j}$ is the electric field density and $A_{k} T$ is the transverse part of the vector potential. The quantities. $r^{k}(t)$ and $p_{k}(t)$ are the canonical variables of the particle. Hence $\widetilde{T}_{M^{0}{ }_{k}}$ is independent of the metric and has the same form as the symmetric stress-tensor's momentum density in flat space. Consequently, it differs from the canonical momentum density,

$$
\boldsymbol{T}_{M}^{C{ }^{\prime}{ }_{k}}=\mathcal{E}^{j T} A_{j}{ }^{T}, k+p_{k}(t) \delta^{3}[\mathbf{r}-\mathbf{r}(t)]
$$

only by a divergence. The proof of this makes use of the fact that $\varepsilon=\varepsilon^{T}+\nabla\left(1 / \nabla^{2}\right) e \delta^{3}$, where $\nabla^{2}$ is the flat space Laplacian operator.

From Eq. (A1), one obtains the extended form of Eq. (3.11) :

$$
-2 \pi_{i, j}{ }^{j}=-\pi^{l m T T} g_{l m}{ }^{T T}{ }_{i}+\mathcal{T}_{M^{0}}{ }_{i}+D_{i j, j},
$$

where $D_{i j, j}$ is the divergence in Eq. (3.11). Hence $-2 \pi_{i}{ }^{j}, j$ differs by a divergence from the total canonical momentum density,

$$
T^{C}{ }_{i}=-\pi^{l m T T_{g_{l m}} T T}{ }_{, i}+T_{M}^{C 0}{ }_{i}
$$

Finally, as was shown in Eq. (3.12), $-2 \pi_{i}{ }^{j}, j$ differs from $-2 \pi^{i j}, j$ by a divergence.

That $-\nabla^{2} g^{T}$ correctly gives rise to time translations in the coupled case, follows immediately from the results of Sec. 2. In $V$, it was shown that the coefficient of $\partial_{t}\left(-\frac{1}{2} \bar{\pi}^{T}\right)$ in the Lagrangian of Eq. (2.18) was unaltered by the presence of matter. Therefore, the discussion of Sec. 2 is completely unchanged by coupling. 